Prätzel-Wolters, D.; Owens, David H.; Ilchmann, Achim :

Robust adaptive stabilization by high gain feedback and switching

Zuerst erschienen in:

International Journal of Control 49 (1989), Nr. 6, S. 1861-1868 


\title{
Robust adaptive stabilization by high gain feedback and switching
}

\author{
D. PRÄTZEL-WOLTERS $\dagger$, D. H. OWENS $\$$ and A. ILCHMANN†
}

A wide class of stabilizing adaptive control rules is investigated. The feedback laws combine high gain adaptive procedures with switching functions and stabilize the systems without knowledge of the sign of the high frequency gain. Furthermore, some non-linear structural perturbations are tolerated.

\section{Introduction}

The problem of stabilizing a linear plant

$$
\dot{y}(t)=a y(t)+b u(t), \quad x(t) \in R, \quad u(t) \in R
$$

with $a$ and $b$ unknown numbers, $b \neq 0$, by a smooth controller of the form

$$
\begin{aligned}
u(t) & =g(y(t), t) \\
\dot{k}(t) & =f(y(t), t)
\end{aligned}
$$

has been considered in a series of papers (Morse 1983, Nussbaum 1983, Willems and Byrnes 1984, Heymann et al. 1985). A variety of stabilizing control rules (1.2), (1.3) is known also for controllable and observable scalar systems $(A, b, c)$ of higher order $n$ which have $(n-1)$ stable zeros and multivariable systems $(A, B, C)$ which are controllable, observable, minimum phase and satisfy det $C B \neq 0$ (cf. Byrnes and Willems 1984, Mårtensson $1986 \mathrm{a}, 1986$ b). All these controllers are combinations of a high gain adaptation law $\dot{k}=f(y, k)$ with some switching devices inherent in $u=$ $g(y, k)$. Willems and Byrnes (1984) prove that the controller:

$$
\begin{aligned}
& \dot{k}(t)=y(t)^{2} \\
& u(t)=s(k(t)) \cdot k(t) \cdot y(t)
\end{aligned}
$$

where $s: R \rightarrow R$ is a switching function bounded on compact sets which satisfies:

$$
\sup _{k \geqslant 1} \frac{1}{k} \int_{0}^{k} s(\sigma) \sigma d \sigma=+\infty \quad \text { and } \quad \inf _{k \geqslant 1} \frac{1}{k} \int_{0}^{k} s(\sigma) \sigma d \sigma=-\infty
$$

globally stabilizes (1.1) in the sense that

$$
\begin{aligned}
& \lim _{t \rightarrow \infty} y(t)=0 \\
& \lim _{t \rightarrow \infty} k(t)=k_{\infty}<\infty
\end{aligned}
$$

In the recent paper of Ilchmann et al. (1987) for MIMO systems

$$
\dot{x}(t)=A x(t)+B u(t)
$$

Received 29 February 1988.

$\dagger$ Institut für Dynamische Systeme, Universität Bremen, 2800 Bremen 33, West Germany.

$\ddagger$ Department of Mechanical and Process Engineering, University of Strathclyde, Glasgow G1 1XJ, Scotland, U.K. 


$$
y(t)=C x(t)
$$

which satisfy:

$$
\begin{aligned}
& (A, B, C) \text { is minimum phase } \\
& \sigma(C B) \subset \mathbb{C}^{-}
\end{aligned}
$$

a large class of adaptation laws (1.3) is generated. They enable the designer to guarantee solutions in any choice of finite intersections of $L_{p}[0, \infty)(p \geqslant 1)$ spaces and are tolerant to 'small' finite gain memoryless non-linearities in state $X$. However here knowledge of the 'sign of the high frequency gain' is assumed to be available $(\sigma(C B)$ $\subset \mathbb{C}^{-}$), hence no switching concepts are necessary.

In this paper we extend these concepts to scalar systems which satisfy (1.10) with $c b \neq 0$. However, the sign of the high frequency gain, sgn $c b$, is not known. We allow switching as a function of both current and past gain and output data and the generalizations permit a wide range of previously unknown gain adaptation mechanisms to be introduced. Furthermore as in the switching free case the controllers are robust with respect to 'small' non-linear structural disturbances.

\section{Stabilization by high gain feedback and switching}

Let $\Sigma\left(\lambda_{0}\right)$ denote the set of controllable and observable scalar systems:

$$
\begin{aligned}
& \dot{x}=A x+b u \\
& y=c x
\end{aligned}
$$

which have $(n-1)$ zeros in the open left half-plane $\mathbb{C}^{-\lambda_{0}}=\left\{s \in \mathbb{C} \mid \operatorname{Re} s<-\hat{\lambda}_{0}\right\}$ and satisfy

$$
c b \neq 0
$$

where $(A, b, c) \in R^{n \times n} \times R^{n \times 1} \times R^{1 \times n}$.

As a generalization of (1.4) we consider the following control law:

$$
u(t)=N(s(t)) \cdot k(t) \cdot y(t), \quad \dot{s}(t)=k(t) \cdot y(t)^{2}, \quad s(0)=s_{0}>0
$$

where:

$$
N: R^{+} \rightarrow R \quad \text { and } \quad k: R^{+} \rightarrow R
$$

are piecewise continuous functions which satisfy the conditions:

$$
\begin{gathered}
\lim _{s \rightarrow \infty} \sup \frac{1}{s} \int_{0}^{s} N(\mu) d \mu=+\infty \\
\lim _{s \rightarrow \infty} \inf \frac{1}{s} \int_{0}^{s} N(\mu) d \mu=-\infty \\
k(t) \geqslant \varepsilon>0 \quad \text { for all } t \in R^{+} \text {and some } \varepsilon>0
\end{gathered}
$$

In order to show that the controller (2.3) stabilizes every system $(A, b, c) \in \Sigma\left(\lambda_{0}\right)$ we need an appendix lemma of Willems and Byrnes (1984). With regard to the next section we state here without proof the following robust version of this lemma. 


\section{Lemma 2.1}

Consider the SIMO finite-dimensional linear system

$$
\begin{aligned}
& \dot{x}(t)=A x(t)+b u(t)+g(x(t), t) \\
& y(t)=C x(t)
\end{aligned}
$$

with $\sigma(A) \subset \mathbb{C}^{-}$and $g: R^{n} \times R \rightarrow R^{n}$ continuous. Then for all $x(0) \in R, T \in R^{+}$and $u(\cdot) \in L_{2}([0, T], R)$ there exist $M_{1}, M_{2}<\infty$ and $\delta>0$ such that

$$
\int_{0}^{T}|u(t)||y(t)| d t \leqslant M_{1}\left\|x_{0}\right\|^{2}+M_{2} \int_{0}^{T}\|u(t)\|^{2} d t
$$

for all $g$ which satisfy the Lipschitz condition

$$
\|g(x(t), t)\| \leqslant \delta \cdot\|x(t)\| \quad \text { for all } t \in R_{+}, \quad x(t) \in R^{n}
$$

The proof of the next lemma is based on the stability proof of Willems and Byrnes for the adaptation law (1.4).

\section{Lemma 2.2}

Let $N, k: R^{+} \rightarrow R$ be piecewise continuous functions satisfying (2.4) and (2.5). Then for all $(A, b, c) \in \Sigma(0)$, all $x(0) \in R^{n}, s(0)>0$ the controller

$$
\begin{aligned}
& u(t)=N(s(t)) k(t) y(t) \\
& \dot{s}(t)=k(t) y(t)^{2}
\end{aligned}
$$

will stabilize $(A, b, c)$ in the sense that

$$
\begin{aligned}
& \lim _{t \rightarrow \infty} y(t)=0 \\
& \lim _{t \rightarrow \infty} s(t)=s_{x}<+\infty \\
& y(\cdot), x(\cdot) \in L_{p}^{n}[0, \infty), \quad 2 \leqslant p \leqslant \infty
\end{aligned}
$$

Proof

Choosing a basis compatible with the state space decomposition $R^{n}=\operatorname{ker} c \oplus \operatorname{im} b$ the system (2.2) can be written as

$$
\left.\begin{array}{c}
{\left[\begin{array}{l}
\dot{x}_{1}(t) \\
\dot{x}_{2}(t)
\end{array}\right]=\left[\begin{array}{ll}
A_{1} & A_{2} \\
A_{3} & A_{4}
\end{array}\right]\left[\begin{array}{l}
x_{1}(t) \\
x_{2}(t)
\end{array}\right]+\left[\begin{array}{l}
0 \\
\beta
\end{array}\right] u(t)} \\
y(t)=\left[\begin{array}{ll}
0 & \gamma
\end{array}\right]\left[\begin{array}{l}
x_{1}(t) \\
x_{2}(t)
\end{array}\right]
\end{array}\right\}
$$

with

$$
\begin{gathered}
\sigma\left(A_{1}\right) \subset \mathbb{C}^{-} \quad \text { (minimum phase properties) } \\
\gamma \cdot \beta=c b \neq 0 \quad \text { (relative degree } 1)
\end{gathered}
$$

Applying the controller (2.3) to (2.10) we obtain:

$$
\dot{x}_{1}(t)=A_{1} x_{1}(t)+A_{2} \gamma^{-1} y(t)
$$




$$
\dot{y}(t)=\left[A_{4}+\gamma \beta N(s(t)) \cdot k(t)\right] y(t)+\gamma A_{3} x_{1}(t)
$$

Thus

$$
\begin{aligned}
\dot{y} y & =[\gamma \beta N(s) k] y^{2}+\gamma A_{3} x_{1} y \\
& =A_{4} \frac{\dot{s}}{k}+\gamma \beta N(s) \dot{s}+\gamma A_{3} x_{1} y
\end{aligned}
$$

and integration yields:

$$
\frac{1}{2} y^{2}(t)-\frac{1}{2} y^{2}(0)=A_{4} \int_{0}^{t} \frac{\dot{s}(\tau)}{k(\tau)} d \tau+\gamma \beta \int_{0}^{t} N(s(\tau)) \dot{s}(\tau) d \tau+\gamma A_{3} \int_{0}^{t} x_{1}(\tau) y(\tau) d \tau
$$

'Change of variables' gives

$$
\int_{0}^{t} N(s(\tau)) \hat{s}(\tau) d \tau=\int_{s(0)}^{s(t)} N(\mu) d \mu
$$

and by Lemma 2.1 applied to $(2.11$ a) we have

$$
\int_{0}^{t}|y(\tau)|\left|x_{1}(\tau)\right| d \tau \leqslant \mu_{1}\left\|x_{1}(0)\right\|^{2}+\mu_{2} \int_{0}^{t} y^{2}(\tau) d \tau
$$

for some $\mu_{1}, \mu_{2}>0$.

Combining (2.13), (2.5) and (2.15) with (2.12) we conclude

$$
\begin{aligned}
\frac{1}{2} y^{2}(t) \leqslant & \frac{1}{2} y^{2}(0)+\frac{\left|A_{4}\right|}{\varepsilon} \cdot[s(t)-s(0)] \\
& +\gamma \beta \int_{s(0)}^{s(g)} N(\mu) d \mu+\mu_{1}\left|\gamma A_{3}\right|\left\|x_{1}(0)\right\|+\mu_{2}\left|\gamma A_{3}\right| \int_{0}^{t} \frac{\dot{s}(\tau)}{k(\tau)} d \tau \\
= & c_{1}+c_{2} s(t)+\gamma \beta \int_{s(0)}^{s(t)} N(\mu) d \mu
\end{aligned}
$$

for some $c_{1} \in R, c_{2} \in R_{+}$.

Because $s(\cdot)$ does not change signs $\left(s(t)>0 \forall t \in R^{+}\right)$it is easily seen that (2.4) implies:

$$
\begin{aligned}
& \lim _{s \rightarrow \infty} \sup \left[\eta s+\xi \int_{s_{0}}^{s} N(\mu) d \mu\right]=+\infty \\
& \lim _{s \rightarrow \infty} \inf \left[\eta s+\xi \int_{s_{0}}^{s} N(\mu) d \mu\right]=-\infty
\end{aligned}
$$

for every $\eta, \xi \in R, \xi \neq 0$.

Since the right-hand side of the inequality (2.14) has to be $\geqslant 0$ we conclude that $s(\cdot)$ has to remain bounded and because $s(\cdot)$ is monotonically not decreasing

$$
\lim _{t \rightarrow \infty} s(t)=s_{\infty}<\infty
$$

Furthermore, because

$$
s(t)=\int_{0}^{t} k(\tau) y(\tau)^{2} d \tau+s(0) \geqslant \varepsilon \int_{0}^{t} y(\tau)^{2}+s(0)
$$


we obtain

$$
y(\cdot) \in L_{2}[0, \infty) \text { and hence } x(\cdot) \in L_{2}^{n}[0, \infty)
$$

because by $(2.10 a) x_{1}(\cdot)$ is the response of a stable system to an $L_{2}$-input signal. But otherwise by $(2.12 a) y(\cdot) \in L_{\infty}[0, \infty)$ and again by $(2.10 a) x(\cdot) \in L_{\infty}^{n}[0, \infty)$. This altogether implies

$$
x(\cdot) \in L_{p}^{n}[0, \infty) \text { for } p \geqslant 2
$$

Finally $\lim _{t \rightarrow \infty} y(t)=0$ is an immediate consequence of $y(\cdot), \dot{y}(\cdot) \in L_{2}[0, \infty)$ and $y(\cdot) \in L_{\infty}^{t \rightarrow \infty}[0, \infty)$.

The above lemma should be regarded as an extension of the Willems and Byrnes controller (1.4), (1.5) which is just the special case obtained from (2.6) by setting:

$$
k(\cdot) \equiv 1, \quad N(s)=N_{0}(s) \cdot s
$$

where $N_{0}(s)$ is any Nussbaum gain (Nussbaum 1983).

The assumptions concerning $k(\cdot)$ in Lemma (2.2) are extremely weak and open an infinite number of possibilities creating new 'gain adaptations'. For example, the feedback strategy makes it natural to regard $k(\cdot)$ as the image of a causal map $\phi$ operating on data records for $s(\cdot), y(\cdot)$ and some measurement vector:

$$
z(t)=F(x(t)) \in R^{q}
$$

where $F: R^{n} \rightarrow R^{q}$ is any linear or non-linear function satisfying

$$
\cdot\|F(x)\| \leqslant F_{0}\|x\|
$$

for some $F_{0}>0$ and all $x \in R^{n}$. maps

Let $\hat{\mathscr{K}}_{\mathrm{s}}$ be defined as the set of adaptive gains $(s(\cdot), y(\cdot), z(\cdot)) \mapsto k(\cdot)$, i.e. causal

$$
\psi: \mathscr{F} \rightarrow L_{\infty}[0, \infty)
$$

where $\mathscr{F}$ is any finite or infinite intersection of product spaces of the form

$$
L_{\infty}[0, \infty) \times L_{r_{1}}[0, \infty) \times L_{r_{2}}^{q}[0, \infty) \text { with } 2 \leqslant r_{i} \leqslant \infty, \quad i=1,2
$$

with the property

$$
k(t)=\psi(s, y, z)(t) \geqslant \varepsilon>0 \text { for all } t \geqslant 0
$$

The following theorem demonstrates that every element of $\hat{\mathscr{K}}_{s}$ together with any switching function of the form (2.4) is able to stabilize every trajectory of a system $(A, b, c) \in \Sigma(0)$ with bounded signals.

\section{Theorem 3}

Let $\psi$ be an element of $\hat{\mathscr{K}}_{s}$ and $N(\cdot)$ be any function satisfying (2.4). Then for all $(A, b, c) \in \Sigma(0)$, all measurements $z(\cdot)$ satisfying (2.18) and all initial conditions $x(0) \in R^{n}, s(0) \in R_{0}^{+}$the controller (2.6) with $k(\cdot)=\psi(s, z, y)$ produces

(i) stable measurements and outputs

$$
z(\cdot) \in L_{p}^{q}[0, \infty), y(\cdot) \in L_{p}[0, \infty) \text { for } p \geqslant 2
$$


(ii) convergent signals $s(\cdot)$

$$
\lim _{t \rightarrow \infty} s(t)=s_{\infty}<\infty
$$

(iii) bounded gains $k(\cdot)$

$$
\exists M>0 \text { such that } k(t) \leqslant M<\infty \text { for all } t \geqslant 0
$$

Proof

(i)-(iii) are immediate consequences of Lemma 2.2, because of (2.21) and (2.18). However then $s(\cdot) \in L_{\infty}[0, \infty), y(\cdot) \in L_{p}[0, \infty)$ and $z(\cdot) \in L_{p}^{q}[0, \infty)$ for $p \geqslant 2$, hence $k(\cdot)=\psi(s, y, z) \in L_{\infty}[0, \infty)$.

\section{Remark 2.4}

Ilchmann et al. (1986) guaranteed the convergence of this gain parameter with additional assumptions for $k(\cdot)$. Here these gains do not necessarily converge. However, if $k(\cdot)$ is constructed due to the following 'differential equation type' adaptation

$$
\dot{k}(t)=y^{2}(t) \varphi_{1}(s(t), y(t))+\|z(t)\|^{2} \varphi_{2}(s(t),\|z(t)\|)
$$

where $k_{0}>0$ and $\varphi_{i}, i=1,2$ are any polynomials (power series) with positive coefficients, then $k(t)>0$ for all $t \in R^{+}$and $k(\cdot)$ converges to a finite gain $k_{\infty}$.

Since decreasing gains $k(t)$ are not excluded the introduced concepts allow in principle possibilities for gain reduction mechanisms and pole placement algorithms.

\section{Robust stabilization}

The adaptive control laws introduced in $\S 2$ are tolerant with respect to structural perturbations (disturbances) and unbounded feedback disturbances of the correct sign. Structural here means, that the perturbations satisfy a Lipschitz type condition. We consider three types of perturbations:

(i) $\dot{x}(t)=A x(t)+g(x(t), t)$

where the map $g: R^{n} \times R_{+} \rightarrow R^{n}$ is continuous and satisfies for some $g_{0}>0$ :

$$
\|g(x, t)\| \leqslant g_{0}\|x\| \quad \text { for all } t \geqslant 0, \quad x \in R^{n}
$$

i.e. time-varying (linear or) non-linear state dependent disturbances of uniformly bounded finite gain.

(ii) $\dot{x}(t)=A x(t)+b[u(t)+h(x(t), y(t), t)]$

where the map $h: R^{n} \times R \times R^{+} \rightarrow R$ is continuous and satisfies for some $h_{1}, h_{2}>0$ :

$$
\|h(x(t), y(t))\| \leqslant h_{1}\|x\|+h_{2}\|y\| \text { for all } x \in R^{n}, y \in R, t \geqslant 0
$$

i.e. time-varying (linear or) non-linear state and output-dependent perturbations of the plant input of bounded growth (caused for example by feedback loops within the process dynamics or non-linear effects in the plant sensor or actuators).

(iii) $\dot{x}(t)=A x(t)+b[u(t)+f(y(t), t)]$ 
where the map $f: R \times R \rightarrow R^{+}$is continuous and satisfies

$$
c b \cdot y(t) \cdot f(y, t) \leqslant 0 \text { for all } y \in R, t \geqslant 0
$$

i.e. possibly unbounded (linear or) non-linear time-varying output-dependent disturbance of the plant input of the 'correct sign'.

For a fixed $(A, b, c) \in \Sigma(0)$ let the class $C(A, b, c, g, h, f)$ denote the set of all nonlinear time varying systems of the form:

$$
\begin{aligned}
\dot{x}(t) & =A x(t)+g(x(t), t)+b(u(t)+h(x(t), y(t), t)+f(y(t), t)) \\
y(t) & =c x(t)
\end{aligned}
$$

where $g, h$ and $f$ satisfy (3.1)-(3.3).

Then the following robustness result holds.

\section{Proposition 3.1}

Let $N, k: R^{+} \rightarrow R$ be piecewise-continuous functions satisfying (2.4) and (2.5). Then for all $(A, b, c) \in \Sigma(0)$, all $x(0) \in R^{n}, s(0)>0$ there exists a real strictly positive number $\delta$ (independent of $N, k, x(0)$ and $s(0)$ ) such that any trajectory, of the system in the class $C(A, b, c, g, h, f)$ with:

$$
g_{0} \leqslant \delta
$$

is stabilized by the controller (2.6) in the sense that (2.7)-(2.9) are satisfied.

\section{Proof}

Applying as in the proof of Lemma 2.2 a suitable coordinate transformation $T$ in the state space we obtain the following description of the transformed closed-loop system:

$$
\begin{aligned}
\dot{x}_{1} & \left.=A_{1} x_{1}+A_{2} \gamma^{-1} y+g_{1}(x(t), t)\right) \\
\dot{y} & =\left[A_{4}+\beta \gamma N(s(t)) \cdot k(t)\right] y+\gamma A_{3} x_{1}+\gamma g_{2}(x(t), t)+\beta(h(x(t), y(t), t)+f(y(t), t))
\end{aligned}
$$

and

$$
\left\|g_{i}(x(t), t)\right\| \leqslant l_{i} g_{0}\|x(t)\|
$$

for some $l_{i}>0$ and all $t \in R, x(t) \in R^{n}$.

Multiplying (3.5 b) by $y(t)$, integrating and applying (3.2), (3.3) and (3.6) we obtain

$$
\begin{aligned}
\frac{1}{2} y(t)^{2} \leqslant & \frac{1}{2} y(0)^{2}+c_{1} \int_{0}^{t} \frac{\dot{s}(\tau)}{k(\tau)} d \tau+c_{2} \int_{s(0)}^{s(t)} N(\mu) d \mu \\
& +c_{2} \int_{0}^{t} h_{1} \cdot\|x(\tau)\|+h_{2} \cdot|y(\tau)||y(\tau)| d \tau \\
& +|\gamma|\left\|A_{3}\right\| \int_{0}^{t}\left\|x_{1}(\tau)\right\||y(\tau)| d \tau \\
& +k_{2} g_{0} \int_{0}^{t}\|x(\tau)\| \cdot\|y(\tau)\| d \tau
\end{aligned}
$$

for some $c_{1}, c_{2} \in \mathbb{R}, c_{1}>0$. 
However by Lemma 2.1 we have for $g_{0}$ sufficiently small:

$$
\int_{0}^{t}\left\|x_{1}(\tau)\right\| \cdot|y(\tau)| d \tau \leqslant M_{1}\left\|x_{1}(0)\right\|^{2}+M_{2} \int_{0}^{t}|y(\tau)|^{2} d \tau
$$

and because $\|x\| \leqslant l_{3}\left(\left\|x_{1}\right\|+\|y\|\right)$ for some $l_{3}>0$ we furthermore get:

$$
\int_{0}^{t}\|x(\tau)\||y(\tau)| \leqslant \tilde{M}_{1}\left\|x_{1}(0)\right\|^{2}+\tilde{M}_{2} \int_{0}^{t}|y(\tau)|^{2} d \tau
$$

Finally, inserting (3.8) and (3.9) in (3.7) we end with an inequality of the form (2.14) (with different constants) and then exactly the same arguments as in the proof of Lemma 2.2 , imply that conditions (2.7) $-(2.9)$ hold for the perturbed system.

\section{Remark 3.2}

As an immediate corollary of Proposition 3.1 we obtain that Theorem 2.2 remains true if the adaptive controller (2.6) is applied to the perturbed system of class $C(A, b, c, f, g, h)$ instead of $(A, b, c) \in \Sigma(0)$ where the disturbances satisfy (3.1)-(3.3) with $g_{0}$ sufficiently small (cf. condition (3.4)).

\section{REFERENCES}

Byrnes, C. I., and Willems, J. C., 1984, Proc. 23rd Conf. on Decision and Control, Las Vegas, pp. 1574-1577.

Heymann, M., Lewis, J. H., and Meyer, G., 1985, Systems Control Lett., 5, 1985.

Ilchmann, A., Owens, D. H., and Prätzel-Wolters, D., 1987, Systems Control Lett., 8, $397-404$

MÅrtensson, B., 1986 a, Adaptive Stabilization. Doctoral Dissertation. Lund Institute of Technology; 1986 b, Systems Control Lett., 6, 87.

MORSE, A. S., 1983, Outils et Modéles Mathématique pour l'Automatique, l'Analyse de Système et le Traitment du Signal, edited by I. D. Landau, pp. 733-740, vol. 3, (Paris: Editions du CNRS).

Nussbaum, R. D., 1983, Systems Control Lett., 3, 243.

Willems, J. C., and Byrnes, C. I., 1984, Global Adaptive Stabilization in the Absence of Information on the Sign of the High Frequency Gain, Lecture Notes in Control and Information Sciences, No. 62 (Berlin: Springer-Verlag). 